\title{
Similarity in Depressive Symptoms in Adolescents' Friendship Dyads: Selection or Socialization?
}

\author{
Matteo Giletta, Ron H. J. Scholte, William J. Burk, Rutger C. M. E. Engels, Junilla K. Larsen, \\ Mitchell J. Prinstein, and Silvia Ciairano \\ Department of Psychology, University of Torino, Torino, Italy, and Behavioural Science Institute, \\ Radboud University Nijmegen, Nijmegen, the Netherlands
}

Behavioural Science Institute, Radboud University Nijmegen

Behavioural Science Institute, Radboud University Nijmegen

Behavioural Science Institute, Radboud University Nijmegen

Behavioural Science Institute, Radboud University Nijmegen

Department of Psychology, University of North Carolina at Chapel Hill

Department of Psychology, University of Torino

\section{Abstract}

This study examined friendship selection and socialization as mechanisms explaining similarity in depressive symptoms in adolescent same-gender best friend dyads. The sample consisted of 1,752 adolescents ( $51 \%$ male) ages $12-16$ years $(M=13.77, S D=0.73)$ forming 487 friend dyads and 389 nonfriend dyads (the nonfriend dyads served as a comparison group). To test our hypothesis, we applied a multigroup actor-partner interdependence model to 3 friendship types that started and ended at different time points during the 2 waves of data collection. Results showed that adolescents reported levels of depressive symptoms at follow-up that were similar to those of their best friends. Socialization processes explained the increase in similarity exclusively in female dyads, whereas no evidence for friendship selection emerged for either male or female dyads. Additional analyses revealed that similarity between friends was particularly evident in the actual best friend dyads (i.e., true best friends), in which evidence for socialization processes emerged for both female and male friend dyads. Findings highlight the importance of examining friendship relations as a potential context for the development of depressive symptoms.

\section{Keywords}

homophily; depressive symptoms; selection; socialization; friendships

Adolescence is a critical period for the onset of depressive symptoms (Angold, Erkanli, Silberg, Eaves, \& Costello, 2002; Wade, Cairney, \& Pevalin, 2002). During this developmental period, adolescents face important developmental tasks, such as differentiating themselves from their parents and creating new social relationships with peers (see e.g., Buhrmester \& Furman, 1987; Harter, 1997). Consequently, negative and stressful peer experiences during adolescence, such as peer rejection and victimization, are

(C) 2011 American Psychological Association

Correspondence concerning this article should be addressed to Matteo Giletta, Department of Psychology, University of Torino, via Verdi 10, 10124, Torino, Italy.m.giletta@ pwo.ru.nl. 
salient predictors of depressive symptoms (Galambos, Leadbeater, \& Barker, 2004; Joiner \& Barnett, 1994; La Greca \& Harrison, 2005; Nolan, Flynn, \& Garber, 2003; Prinstein, Boergers, \& Vernberg, 2001; Vernberg, 1990). On the other hand, positive and supportive peer relationships have been found to be essential for developing personal skills and thus are likely to contribute to adolescent psychological well-being (see e.g., Berndt, 1989; Bukowski, Newcomb, \& Hartup, 1996; La Greca \& Harrison, 2005).

Yet, adolescents with close and supportive friendship relationships may nevertheless suffer from depressive symptoms. This may be due to a process referred to as depression contagion. Specifically, prior work has theorized that depression may be similar between friends because (a) adolescents select friends who initially are similar to them in their level of depressive symptoms (i.e., referred to as homophilic selection) and/or (b) adolescents' friends' depressive symptoms may exacerbate their own depressive symptoms (i.e., referred to as socialization). Although some past research has suggested that friends tend to be similar in their level of depressive symptoms over time (Haselager, Hartup, van Lieshout, \& Riksen-Walraven, 1998; Hogue \& Steinberg, 1995; Prinstein, 2007; Stevens \& Prinstein, 2005; Van Zalk, Kerr, Branje, Stattin, \& Meeus, 2010), several issues require further investigation to stringently examine the depression contagion hypothesis.

First, previous studies have predominantly examined socialization and paid less attention to homophilic selection mechanisms (see e.g., Prinstein, 2007; Stevens \& Prinstein, 2005). Second, although homophily is a dyadic construct and thus requires investigating a dyadic approach (Popp, Laursen, Kerr, Stattin, \& Burk, 2008), previous studies have mainly examined similarities between adolescents and their friends from an individualistic perspective. Third, studies need to consider the role of different types of friendship. Most previous research has examined only best friends in class or school and has not examined whether these best friends represented the adolescents' true best friends regardless of the context. Prior work has seldom examined whether socialization processes may differ for different friendship types (see e.g., Van Zalk et al., 2010). Therefore, we applied a longitudinal dyadic analytic approach to examine homophily in depressive symptoms in reciprocated best friend dyads, distinguishing between class best friends and true best friends.

\section{Selection and Socialization Processes Related to Depressive Symptoms}

Both selection and socialization mechanisms may contribute to similarities found among adolescent friend behaviors (see e.g., Engels, Knibbe, Drop, \& de Haan, 1997; Ennett \& Bauman, 1994; Kandel, 1978). On the one hand, theories of interpersonal attraction (Byrne, 1971; Kelley \& Thibaut, 1978) suggest that people prefer, and are more attracted to, people with similar attitudes and/or behaviors. Thus, adolescents are more likely to select similar peers as friends; this is known as homophilic selection. Experimental studies have provided evidence that depressed people are more attracted to other depressed people (see e.g., Coates \& Winston, 1983; Joiner, 1994; Rosenblatt \& Greenberg, 1991; Strack \& Coyne, 1983). Consequently, adolescents with certain levels of depressive symptoms may be more likely to prefer and ultimately select adolescents with similar levels of depressive symptoms as friends. On the other hand, during the course of a friendship, adolescents may also influence each other, becoming more similar; this has been referred to as socialization.

Together, these processes may pose unique risks for adolescents with high levels of depressive symptoms. A cyclical process (Coyne, 1976) suggests that adolescents with high levels of depressive symptoms may affiliate with one another, and these relationships may maintain or exacerbate the depressive symptoms of each adolescent (Rosenblatt \& Greenberg, 1991). However, this idea has rarely been tested in previous developmental 
studies, and the effects of friendship selection on adolescent depressive symptoms have seldom been examined. Hogue and Steinberg (1995) investigated both homophilic socialization and selection mechanisms related to internalized distress in stable adolescent friendships. They found evidence for socialization processes among male adolescents who tended to internalize distress to be more similar to the behaviors of peer clique members. This was not the case for female adolescents. Further, Stevens and Prinstein (2005) and Prinstein (2007) showed that the best friend's depressive symptoms predict changes in adolescent depressive symptoms over time (i.e., socialization) while controlling for the initial level of similarity between dyad members. However, because they assessed friendship ties only at the baseline, without considering whether adolescents were still friends at the follow-up, they were not able to provide clear evidence concerning the relative importance of selection versus socialization processes. These studies failed to examine selection and socialization processes because they did not distinguish new friendships established during the course of a study from old friendships established prior to the course of a study that remained stable over time. Importantly, to estimate selection effects, one must consider similarities between adolescents prior to the establishment of the friendship (Kandel, 1978; Popp et al., 2008).

Recently, Van Zalk et al. (2010) dealt with these limitations by applying actor-based models (i.e., models for the coevolution of social networks and individual behavior; Snijders, Steglich, \& Schweinberger, 2007) to investigate depressive symptoms within friend social networks. They found that adolescents selected their friends on the basis of similarities in depressive symptoms. Moreover, they showed that adolescents and their friends became more similar over time by increasing their depressive symptoms. These findings suggest that changes in levels of depressive symptoms and friendship formations may be strongly interrelated during adolescence. However, because this study focused on the social networks of friends, including different types of friendships (i.e., dyadic and triadic friendships, both unilateral and reciprocal), it is not clear whether the same processes take place specifically within some dyadic relations (i.e., best friend dyads). The importance of examining best friend dyads is based on the close and intimate nature of these relationships. Indeed, because adolescents who are involved in these dyadic relationships are likely to share positive or negative emotions (see e.g., Rose, 2002), they may be more likely to show similar levels of depressive symptoms. Moreover, socialization processes related to depressive symptoms may occur through co-rumination, particularly within friend dyads. The excessive and repeated sharing of personal problems and negative feelings between two close friends may contribute to increases in depressive symptoms (Rose, 2002; Rose, Carlson, \& Waller, 2007).

Thus, in the current study we focused, on the basis of this literature, exclusively on best friend dyads. We specifically focused on reciprocated friend dyads because it can be assumed that reciprocated friendships are more intimate than unilateral friendships, and previous research has suggested that reciprocated best friends share higher levels of similarities compared with unilateral friends (see e.g., Haselager et al., 1998). Utilizing a longitudinal design, we differentiated stable from newly established friend dyads in order to examine homophilic friendship selection and socialization, respectively. It was possible to examine selection effects in adolescents who were involved in a best friendship at Time 2 but not Time 1 (i.e., new friendships) as well as socialization effects in adolescents that were best friends at both time points (i.e., enduring friendships). We assumed, on the basis of previous studies (Haselager et al., 1998; Hogue \& Steinberg, 1995; Prinstein, 2007; Stevens $\&$ Prinstein, 2005), similarities in depressive symptoms in reciprocated friendships. We expected both selection and socialization to explain similarities in depressive symptoms in both male and female dyads. Moreover, we also examined gender differences, because previous research has revealed higher friendship intimacy, higher depressive symptoms, and 
stronger depression socialization effects among female adolescents (see e.g., Calmes \& Roberts, 2008; Rose et al., 2007). We expected members of female best friend dyads to report a higher level of similarity in depressive symptoms as well as higher socialization and selection processes compared with male best friend dyads.

\section{Class Best Friends Versus True Best Friends}

Studies on friendship relations have usually focused on best friends in the school context. A common procedure of identifying best friendships consists of asking adolescents to nominate their best friends within their class (see e.g., Adams, Bukowski, \& Bagwell, 2005; Haselager et al., 1998; Poulin \& Boivin, 2000; Rose, 2002), grade (see e.g., Cillessen, Jiang, West, \& Laszkowski, 2005; Prinstein, 2007), or school (see e.g., Hogue \& Steinberg, 1995). In adolescence, however, youths have best friends outside their class as well (see Vandell, Pierce, \& Dadisman, 2005), and thus restricting friendship nominations to other students in the same class or school when examining the role of best friends may lead to inconclusive findings, especially when the best friends in the school context do not represent the adolescents' true best friends.

Best classroom friends might be significantly different from true best friends (i.e., across classroom and nonclassroom contexts). Moreover, it can be assumed that adolescents may have particularly intimate and close relationships with their true best friends (i.e., their closest friends, including those outside of their class). Because close and intimate friendships are likely to be the most influential, similarity in and socialization related to depressive symptoms may occur especially within such relationships (Rose, 2002; Steven \& Prinstein, 2005).

In line with previous friendship literature, adolescents' friendships in this study were identified within the school context. However, we addressed this limitation by assessing whether the classmates whom the adolescents nominated as their best friends represented their true best friend (across all contexts). Here we defined a true best friend as the classmate who would have been nominated as the very best friend even without classroom restrictions associated with the peer nomination procedure. On the other hand, a class best friend was defined as an adolescent's best friend within the class. Thus, a further aim of this study was to test separately similarity in and socialization relevant to depressive symptoms in both a subsample of class best friends and a sub-sample of true best friends. We expected, on the basis of previous literature, higher similarity as well as higher socialization between true best friends dyads compared with class best friends dyads.

\section{A Dyadic Approach to Study Similarity Between Adolescent Friends}

Within close relationships, individuals share feelings, thoughts, and experiences and thus are interdependent. Interdependent relations consequently generate statistical interdependencies (Laursen, Popp, Burk, Kerr, \& Stattin, 2008). Thus, traditional statistical techniques, which assume that individual observations are independent, may not be appropriate to study such relationships (Kenny, 1995). Similarly, studying similarities and interpersonal processes in friend dyads implies correlations between individual scores (Cook \& Kenny, 2005; Popp et al., 2008). Most previous studies on similarity in depressive symptoms among friends did not account for interdependence between the friendship members and thus for statistical dependencies in the data (Hogue \& Steinberg, 1995; Prinstein, 2007; Stevens \& Prinstein, 2005). Disregarding interdependence has two shortcomings. First, the violation of the independent assumption may induce systematic bias in significance tests. That is, the correlated nature of measures among friends artificially reduces the standard errors of parameter estimates, which inflates Type I error. Second, interpersonal influence between individuals may not be estimated. Indeed, traditional statistical methods allow for assessing 
only the influence of one dyad member on the other, whereas in reality friends might mutually influence each other (Laursen et al., 2008).

The application of a longitudinal actor-partner interdependence model (APIM; Cook \& Kenny, 2005; Kashy \& Kenny, 2000; Olsen \& Kenny, 2006) to the study of socialization and selection within dyad members represents one alternative to deal with interdependent data. The APIM allows for simultaneous estimation of the effects of each individual predictor on his or her own outcome variable (i.e., actor effects) and on the partner's outcome variable (i.e., partner effects). Thus, both one's own and one's partner's depressive symptoms at Time 1 may predict both dyad members' depressive symptoms at Time 2 . In the current study, such a model was applied to investigate homophilic mechanisms that might explain depressive symptoms within adolescent friendship dyads.

\section{Method}

\section{Participants}

The sample consisted of 1,752 adolescents ( $51 \%$ male), forming 487 friend dyads ( $n=974$ ) and 389 nonfriend dyads $(n=778)$; the nonfriend dyads served as a comparison group. Ages ranged between 12 and 16 years $(M=13.77, S D=0.73)$, with most $(87.7 \%)$ participants being between 13 and 15 . Participants were recruited from seven secondary schools located in urban and suburban areas of the Netherlands. All adolescents followed regular secondary education; that is, $24.4 \%$ of them were in the first grade, and $75.6 \%$ in the second (i.e., Grades 7 and 8 in the United States, respectively). Specifically, 19.4\% of adolescents attended the lowest education track (43.3\% in the national population), $20.7 \%$ attended intermediate educational tracks ( $26.2 \%$ in the national population), $24.5 \%$ attended intermediate to high educational tracks (18.3\% in the national population), and $35.4 \%$ followed the highest (preuniversity) educational track (12.2\% in the national population). Compared with the national population of this age cohort (CBS, 2006), our sample included a relatively high percentage of adolescents who followed a high level of secondary education. In the Dutch school system, students are assigned to classes in which they spend most of their time and perform most of the school activities (i.e., homeroom). Participants in our sample were from 90 classes (on average 13 for each school), with an average size of approximately 25 students per class. At the beginning of the study, $90.7 \%$ of the adolescents were living with both parents in intact, nondivorced families. Most participants were born in the Netherlands (95.5\%) and had at least one parent who was born in the Netherlands or in another European country (93.4\%). Approximately $11 \%$ of the participants were ethnic minorities from Turkey (2\%), Surinam and Dutch Antilles (1.7\%), Morocco (1\%), or elsewhere (6.3\%).

\section{Measures}

Adolescents completed questionnaires in their classes during regular school hours. Research assistants administered identical surveys at an initial time point (Time 1) and then again 1 year later (Time 2).

Depressive symptoms-Participants completed the Dutch version of the Center for Epidemiological Studies-Depression scale (CES-D; Bouma, Ranchor, Sanderman, \& Sonderen, 1995), which consists of 20 items describing depressive symptoms during the previous week. Items are rated on a scale ranging from 0 (rarely or none of the time) to 3 (most or all the time), with a total score ranging between 0 (no depressive symptoms) and 60 (many depressive symptoms). The psychometric properties of the CES-D have been well documented in various adolescent and adult samples (Cuijpers, Boluijt, \& van Straten, 2008; Garrison, Addy, Jackson, McKeown, \& Waller, 1991; Roberts, Lewinsohn, \& Seeley, 
1991). Satisfactory internal consistency of the scales were observed at both time points ( $a$ $=.86$ and .87 , respectively, for Time 1 and Time 2 ).

Friendship nominations-To identify adolescent friendships, we used rosters of students' root classmates (i.e., students in the same homeroom). Specifically, adolescents were provided with a list of their classmates' names and were asked to nominate up to five best friends in their root class. Adolescents were instructed to rank their class friends in order (i.e., starting with their very best friend, followed by their second best friend, etc.). On average, adolescents selected their friends from a pool of approximately 25 students, and they nominated $4.02(S D=1.4)$ friends at Time 1 and $3.97(S D=1.4)$ friends at Time 2. Only the first nominations (i.e., very best friendships) were considered in the present study, which is in line with previous studies on friendships (see e.g., Parker \& Asher, 1993; Popp et al., 2008; Prinstein, 2007; Rose, 2002). On average in each class, approximately 95\% of the adolescents nominated a very best friend, irrespective of class size. Specifically, a logistic regression analysis showed that class size was not related to the probability of selecting the best friend. Moreover, chi-square analyses identified no differences across grades and schools in the percentage of adolescents who selected a very best friend.

Two additional questions (i.e., "Do you have a best friend?" and "Is your best friend in your same class?") were used to identify whether participants had a true best friend and whether the person attended the same class. Subsequently, participants who reported having a true best friend within their class were asked to nominate the person from the list of their classmates.

\section{Procedures}

Participants in the present study were drawn from the first two waves of a large-scale longitudinal project examining mental health and health habits among Dutch youths (see also Larsen, Otten, \& Engels, 2009). Students were informed that participation was voluntary and confidential. Parents were informed about the study through the mail and were asked to respond via telephone or e-mail if they did not want their child to participate in the study. Of the 2,216 targeted students, $92.6 \%(n=2,051)$ initially participated, and $85.5 \%$ of these participants $(n=1,753)$ also completed surveys 1 year later, a precondition for inclusion in the present study. A logistic regression analysis tested whether adolescents lost at follow-up differed from adolescents who completed surveys at both time points on sociodemographic characteristics (i.e., gender, age, ethnicity, educational level, and family structure) and level of depressive symptoms at both time points. Significant differences emerged on several characteristics. This analysis revealed that gender $(O R=0.70, p<.01$, $95 \%$ CI $[0.54,0.91])$, age $(O R=0.75, p<.01,[0.63,0.91])$, ethnicity $(O R=0.53, p<.001$, $[0.38,0.73])$, family structure $(O R=1.50, p<.05,[1.07,2.10])$, educational level $(O R=$ $1.13, p<.05,[1.01,1.27])$, and depressive symptoms $(O R=0.97, p<.001,[0.95,0.98])$ significantly predicted attrition. That is, female adolescents, younger adolescents, those of Dutch origin, those living with two biological parents, and those with lower levels of depression were overrepresented in the longitudinal sample.

In the longitudinal sample, adolescents were considered members of a best friendship dyad when the classmate they nominated as a very best friend (i.e., their first friend listed) reciprocated the nomination as a very best friend at least at one time point. Because $18.2 \%$ $(n=320)$ of participants were not involved in a reciprocal friendship at any time point, they were not included in friend dyads. On the contrary, some participants were involved in reciprocated very best friendships with different friends at the two waves. The inclusion of all friendship dyads in the analyses would have violated the independence assumption of multiple-group modeling because of introducing potential bias due to unequal contributions 
by individuals. Thus, to avoid these problems, and in line with previous studies that adopted similar methodologies (Popp et al., 2008; Prinstein, 2007), we restricted each participant to only one friendship dyad, by randomly selecting the friendship at Time 1 (i.e., dissolved friendship) or at Time 2 (i.e., new friendship). As a result, another $24.4 \%(n=427)$ of adolescents were excluded, reducing our sample to 1,006 participants, corresponding to 503 best friend dyads. Only seven dyads were of mixed gender. These were excluded from further analyses. In addition, nine students provided incomplete data and were therefore omitted from analyses, reducing the analytic sample to 487 same-gender best friendship dyads (252 female dyads). A logistic regression analysis showed no significant differences between adolescents who were included and excluded in friend dyads in the level of depressive symptoms and most of the sociodemographic characteristics (i.e., age, ethnicity, educational level, and family structure). A significant effect emerged only for gender ( $O R=$ $0.76, p<.01,95 \% \mathrm{CI}[0.62,0.93])$, indicating that female adolescents were overrepresented in friend dyads. ${ }^{1}$ Thus, overall, the analytic sample was representative of the entire longitudinal sample.

The 487 best friendship dyads were classified into three groups that differed in the initiation and duration of the relationship. Dissolved friendships described relationships in which both individuals nominated each other as best friends at Time 1 but not at Time 2 (93 male dyads and 84 female dyads). New friendships described relationships in which both individuals nominated each other as best friends at Time 2 ( 85 male dyads and 95 female dyads) but not at Time 1. Enduring friendships described relationships in which both individuals nominated each other as best friends at both time points (57 male dyads and 73 female dyads). A chisquare analysis revealed no gender differences in these three types of friendships, $\chi^{2}(2)=$ $2.39, p=.30$.

In addition, to test our final hypothesis, we further classified enduring friendship dyads into two groups: true best friends ( $n=44 ; 17$ male dyads and 27 female dyads) and class best friends ( $n=86 ; 40$ male dyads and 46 female dyads). True best friend dyads described friendships in which both adolescents nominated each other as true best friends, whereas best friends in class described friendships in which both adolescents nominated each other as best friends in class but not outside the class. No gender differences emerged in the size of the two subsamples, $\chi^{2}(1)=0.73, p=.39$, indicating that the probability of having a true best friend who attended the same class was similar for male and female adolescents.

Finally, we selected, in line with studies on friend similarities (see e.g., Popp et al., 2008; Poulin \& Boivin, 2000), a comparison group of nonfriend dyads consisting of adolescents who were present at both time points but who were excluded from friend dyads. This comparison group was used to test whether the similarities between friends differed significantly from similarities between nonfriends in order to demonstrate that similarity in depressive symptoms was unique to friends. The dyads in this group consisted of randomly paired adolescents who did not nominate one another as friends at either time point. The comparison group included 389 same-gender and same-age dyads (179 female dyads). The adolescents selected for the comparison group did not differ significantly from the adolescents in the friend dyads on any of the study variables (see the results of the previously mentioned logistic regression analysis).

\footnotetext{
${ }^{1}$ Note that because all the analyses were conducted separately by gender, the inclusion of more female adolescents in the analytic sample did not affect our results.
} 


\section{Plan of Analysis}

We initially assessed, as an essential condition for dyadic analyses, the degree of similarity (i.e., interdependence) between friends' outcome variables (i.e., depressive symptoms at Time 2). Because best friends in dyads had the same characteristics in terms of age and gender in our sample, it was not possible to differentiate their role in the relationship (see also Cillessen et al., 2005). Thus, we did not distinguish dyad members. Consequently, the degree of dyadic similarity on depressive symptoms was assessed with intraclass correlations (ICCs; Kenny, Kashy, \& Cook, 2006). ICCs were computed separately for male and female dyads in the new and enduring friendship groups. ICCs were also calculated for the nonfriend comparison group. ICC contrasts were used to compare similarities of friends in friendship dyads with similarities of adolescents in nonfriend dyads.

To examine selection and socialization processes related to depressive symptoms, we performed a series of longitudinal APIM models for indistinguishable dyads (Kenny et al., 2006), applying the multiple-group strategy employed by Popp et al. (2008). APIM models consist of actor effects, which represent individual stability paths, and partner effects, which represent paths of interpersonal influence and thus provide evidence for socialization effects. Therefore, the effects of friend depressive symptoms at Time 1 on adolescent depressive symptoms at Time 2 (i.e., partner effects) were estimated while controlling for adolescent depressive symptoms at Time 1 (i.e., actor effects). Additionally, these models provide concurrent correlations between the independent variables (i.e., depressive symptoms at Time 1) as well as between the residuals in the dependent variables (i.e., depressive symptoms at Time 2). Thus, actor and partner effects were estimated simultaneously while adjusting for the initial correlations between friends' reports (see Figure 1).

The concurrent correlation at Time 1 represents a measure of initial similarity between dyad members. Thus, it may provide evidence for the selection effects in dyads consisting of friends who were not best friends at Time 1 but became best friends at Time 2 (i.e., new friendships). Moreover, in longitudinal models, the correlation between residuals may be interpreted as a measure of increased dyadic similarity over time (Laursen et al., 2008). Thus, a significant correlation between residuals in dyads consisting of friends who were best friends at both time points (i.e., enduring friendships) provides further evidence for socialization effects. For the enduring friendship group, we also calculated ICC proportions; that is, the part of the correlation between the dependent variables that may be attributable to the socialization processes and individual stability (see Kenny et al., 2006, p. 146).

Additional ICCs and APIM models were analyzed to investigate differences between the two subsamples of the enduring friendships: class best friends and true best friends dyads. Adolescents who were best friends only at Time 1 but not at Time 2 (i.e., dissolved friendships) were used exclusively for comparative purposes.

Specific considerations need to be made for the APIM models for indistinguishable dyads (see Olsen \& Kenny, 2006). Specifically, the means, intercepts, variances, residual variances, actor effects, and partner effects are forced to be identical for both members of a dyad (see Figure 1). These modeling constraints were necessary to eliminate differences associated with the random assignment of some youths into a target group and some youths into a friends group. For these analyses, we used maximum-likelihood estimation with the Huber-White covariance adjustment (MLR in Mplus 5.0; Muthén \& Muthén, 1998-2007). To compare estimates between models, we specified equality constraints and tested the differences between models using the adjusted chi-square difference test specified by Satorra and Bentler (2001). 


\section{Results \\ Descriptive Analyses}

We performed a 3 (friendship group) $\times 2$ (gender) $\times 2$ (time) repeated-measure analysis of variance to examine mean-level gender and friendship group differences on participants' depressive symptoms. A main effect emerged only for gender, $F(1,968)=79.71, p<.001$, indicating that within each friendship group, female adolescents reported a higher level of depressive symptoms compared with male adolescents at both time points.

Table 1 presents the means and standard deviations for depressive symptoms separately by gender and friendship groups.

\section{Similarity Between Best Friends' Reports of Depressive Symptoms at Time2}

Best friends' homogeneity on depressive symptoms at Time 2 emerged for both female and male adolescents in new friendships. The ICC values showed a small degree of similarity between dyad members $\left(r^{2}=.16\right.$ and $.19, p<.05$, respectively, for female and male adolescents). In enduring friendships, a modest degree of similarity in depressive symptoms was found for female dyads $\left(r^{2}=.40, p<.01\right)$ but not for male dyads $\left(r^{2}=.02, p=.43\right)$. ICC contrasts (Kenny et al., 2006) were used to compare the magnitude of ICCs for male and female friendship dyads. Significant gender differences emerged regarding enduring friendships, indicating that members of female dyads reported significantly higher levels of similarity in depressive symptoms compared with male dyad members $(z=2.47 ; p=.007)$.

The ICCs of the nonfriend comparison group were not significant for female or male adolescents $\left(r^{2}=.01\right.$ and -.01 , respectively). ICC contrasts showed a difference in the ICC values between the enduring female friendships and the nonfriend female group $(z=2.94 ; p$ $=.003$ ), suggesting that similarity within the best friend dyads was significantly higher compared with similarity between nonfriends. No differences in the ICC values emerged between the new friendships and the nonfriend group for either male or female adolescents at Time 2 .

Overall, these results suggest that similarity in depressive symptoms is unique to members of friend dyads. However, even if the ICCs were statistically significant, the absence of a significant difference between the new friendship groups and the comparison group indicate that members of new friendships were only slightly similar in their level of depressive symptoms. The finding signifying significant correlations between partners' dependent variables had an important implication for the subsequent dyadic analyses. Dyadic interdependence is a prerequisite for APIM models (Kenny et al., 2006), yet we found evidence of interdependence only for female friendship groups and the male new friendship group. However, for comparative purposes, we also present results for the male enduring friendships as well as for dissolved friendships.

\section{Longitudinal APIM Models}

We first tested whether a constraint model in which male and female adolescents formed a single model of depressive symptoms would fit the data. This model did not fit the data well (comparative fit index $[\mathrm{CFI}]=.429$; root-mean-square error of approximation $[\mathrm{RMSEA}]=$. 000; Tucker-Lewis index $[\mathrm{TLI}]=.689$ ). Moreover, the chi-square difference test showed that these constraints significantly worsened the fit of the model to the observed data, $\Delta \chi^{2}(8)=102.98, p=.001$, suggesting gender differences within the model.

Subsequently, we tested whether the three friendship groups (i.e., dissolved friendships, new friendships, enduring friendships) of male and female adolescents could be constrained to 
form a single group. Again, the constrained model did not fit the data well (CFI = .841; RMSEA $=.000 ; \mathrm{TLI}=.919)$ and the chi-square difference test significantly worsened the fit of the model to the data, $\Delta \chi^{2}(32)=46.13, p=.050$. This implies significant differences between the three friendship groups (i.e., dissolved, new, or enduring friendship dyads). Each of these friendship dyad groups was examined separately for male and female adolescents (see Table 2).

Selection effects related to depressive symptoms-To identify selection effects, we examined similarity in the levels of depressive symptoms at Time 1 between new friendships. One might assume selection processes if adolescents who reported similar levels of depressive symptoms at Time 1 subsequently selected each other as best friends at Time 2 .

A measure of similarity in depressive symptoms was provided in the APIM model by the correlations between depressive symptoms of dyad members. Overall, for new friendships, the correlations between depressive symptoms at Time 1 indicated the absence of significant homophilic selection effects in both male and female best friend dyads (see Table 2).

Specifically, in female dyads, a significant correlation was negative, indicating the tendency among female adolescents to select as friends those with a different level of depressive symptoms.

Significant concurrent correlations emerged for some of the other friendship groups (see Table 2). However, the absence of information about depressive symptoms before the establishment of the friendships did not allow us to know whether these similarities arose from selection or socialization processes.

Socialization effects related to depressive symptoms-In our sample, we could detect socialization effects only in enduring friendships. Socialization effects would occur when adolescents who were already best friends at Time 1 affected each other's depressive symptoms over time. In each of the APIM models, the actor effects emerged as statistically significant $(p<.001)$, indicating that adolescent reports of depressive symptoms are moderately stable over 1 year. However, partner effects emerged as statistically significant only for females in enduring friendships (see Table 2). These results provided evidence for socialization in female adolescents' dyads, indicating that female adolescents' depressive symptoms predicted increases in their best friends' depressive symptoms over time. No partner effects were found for male adolescents in enduring friendships. We tested an additional set of equality constraints to investigate the relative magnitude of the partner effects in the female enduring friendship dyads. This set of constraints compared partner effects in the female enduring friendships with partner effects in the other five friendship groups. These constraints worsened model fit, $\Delta \chi^{2}(1)=4.31, p=.04$, indicating that the partner effects for the female enduring friendship dyads were significantly higher in magnitude compared with the other five friendship groups.

Moreover, a significant correlation between residuals emerged for female adolescents in enduring friendships, suggesting that members of these female dyads tended to become more similar over time (see Table 2). ICCs indicated that individual stability and dyadic influences explain $23 \%$ of this similarity. No significant residual similarity emerged for the male adolescents in enduring friendships. A significant correlation between residuals emerged also for female adolescents in new friendships (see Table 2). However, because these dyad members were not best friends at Time 1, such similarity could not be interpreted as the result of socialization processes. 


\section{Similarity and Socialization in Class Best Friends and True Best Friends}

To differentiate between best friends in class and true best friends, we calculated ICCs at Time 2 for both types of very best friends. These ICCs were significant for the two female subsamples, $r^{2}=.27, p<.05$ and $r^{2}=.63, p<.001$, for class best friends and true best friends, respectively. For male adolescents, a significant ICC emerged only for the true best friend group $\left(r^{2}=.44, p<.05\right)$, whereas the ICC between male class best friends was nonsignificant $\left(r^{2}=-.17\right)$. ICC contrasts were computed separately for female and male adolescents to compare the ICC values between the true best friends and class best friends. Differences were significant in male dyads $(z=2.11 ; p=.003)$, and they approached significance in female dyads $(z=1.85 ; p=.063)$. These results showed that in the enduring friendship dyads, members of true best friend dyads tended to report higher levels of similarity compared with the members of class best friend dyads.

An additional series of multigroup APIM models were conducted to estimate socialization effects in both male and female enduring friendships in the class best friend and the true best friend groups. A chi-square difference test showed that a model with equality constraints applied to all four groups significantly worsened model fit, $\Delta \chi^{2}(24)=48.29, p=.002$. Thus, Table 3 reports the actor and partner effects separately for each group.

Significant actor effects emerged for male and female adolescents in both the enduring friendship dyads, showing individual stability in depressive symptoms over time. In addition, significant partner effects in all groups indicated that friends' levels of depressive symptoms predicted adolescents' depressive symptoms over time. However, the partner effect for the male class best friend dyads was negative. That is, for these male adolescents, higher levels of depressive symptoms predicted decreases in the best friend's depressive symptoms over time (see Table 3). Moreover a positive significant correlation between residuals emerged exclusively for the female true best friends (see Table 3), indicating that members of these dyads tended to become more similar in depressive symptoms over time. ICC proportions indicated that individual stability and dyadic influences explain $23 \%$ of this similarity. The correlations between residuals for other groups suggested that friends within these dyads did not significantly increase their similarity over time. On the contrary, the negative and significant correlation for the male class best friend group suggested that male adolescents in this group became less similar over time.

In sum, these additional findings suggested differences between class best friends and true best friends in socialization processes. For female adolescents, even if partner effects were identified in both groups, such effects contributed significantly to an increase in friends' similarity over time only among true best friends. For male adolescents in class best friend dyads, the negative partner effect and correlation of residuals suggested that members of these dyads influenced each other's depressive symptoms in opposite directions, increasing their dissimilarity over time. Nevertheless, significant partner effect for the members of true best friend dyads indicated positive socialization processes.

Reanalyzing the APIM models (see Table 2 and 3) while controlling for adolescents' age and educational level revealed equivalent results with highly similar standardized estimates.

\section{Discussion}

The present study aimed to explore similarity in depressive symptoms between adolescents and their best friends and to examine the homophilic mechanisms that might explain such similarity (i.e., selection and socialization). Similarity and socialization processes were also examined within two types of friendship dyads: class best friends and true best friends. We extended past research on homophily by applying a dyadic approach designed for the study 
of close relationships with interdependent data. Moreover, the identification of friendships that started and ended at different time points allowed us to clearly identify selection and socialization processes. Overall, the results partially confirm our hypothesis, indicating that similarity in depressive symptoms exists exclusively in female dyads. In particular, we found that socialization processes explained this similarity in female friendships.

With respect to the first aim, our findings provided support for the general interpersonal similarity-attraction principle (see McPherson, Smith-Lovin, \& Cook, 2001), because adolescents tended to report levels of depressive symptoms that were similar to their best friends'. Although research has indicated that friends are similar in externalizing behaviors (see e.g., Engels et al., 1997; Ennett \& Bauman, 1994; Kandel, 1978), only a few studies showed homogeneity in internalizing behaviors within friendships (Haselager et al., 1998; Hogue \& Steinberg, 1995; Prinstein, 2007; Stevens \& Prinstein, 2005; Van Zalk et al., 2010). Our findings suggest that internalizing behaviors might also play a salient role in the formation of adolescent friendships. Such similarity in depressive symptoms was especially evident within female dyads. This gender-specific similarity can be explained by the fact that female adolescents, more so than male adolescents, are likely to report high levels of internalizing problems, and at the same time, they are more likely to form close and intimate relationships in which they might discuss such problems (see e.g., Rose, 2002). Thus, internalizing behaviors might represent a more salient feature in female friendships, whereas externalizing behaviors might play a more central role in male friendships (Dishion, Andrews, \& Crosby, 1995; Poulin \& Boivin, 2000).

The second aim was to examine the relative importance of friendship selection and socialization in relation to depressive symptoms. Compared with previous findings, our findings for the different types of friendships allowed us to obtain a more detailed picture of the role of socialization and selection. Concerning selection processes, the absence of similarity in both male and female new friendship dyads at baseline indicates that homophilic selection did not explain similarities between friends in depressive symptoms at follow-up. In contrast to the results of previous studies (Hogue \& Steinberg, 1995; Van Zalk et al., 2010), this finding suggests that similarities in depressive symptoms do not seem to influence friendship formation. This difference might be because whereas previous studies investigated selection either in cliques or in social networks, we specifically focused on reciprocated best friend dyads. Other peer characteristics may explain why certain adolescents select some peers but not others to become their best friends. For instance, examining best friend dyads, Popp et al. (2008) showed that adolescents selected as friends peers with similar levels of alcohol intoxication.

Our results not only failed to provide support for homophilic selection mechanisms (i.e., friends' selection based on similar characteristics) but also seemed to suggest the opposite; that is, the tendency among female adolescents to select as friends peers with a different level of depressive symptoms. One might speculate that female adolescents with higher levels of depressive symptoms may seek out reassurance or social support specifically from friends with lower levels of depressive symptoms, because this may boost their own psychosocial well-being (Coyne, 1976). Considering that female adolescents perceive more social support from friends than do male adolescents (see e.g., Colarossi \& Eccles, 2003; Slavin \& Rainer, 1990), it is not surprising that such a selection process emerged only for female friendships. However, given the small effect size, this result needs to be interpreted with caution.

The analyses concerning the enduring friendship group revealed that female friends affected each other's depressive symptoms over time. This finding suggests that socialization processes may explain similarity in depression. However, such processes are likely to be 
absent in male friends, because our findings did not show partner effects over time in these dyads. These contrasting findings may be explained by the different roles that friendships play in female compared with male dyads. Female adolescentst end to engage in more close and intimate dyadic relationships in which they are more likely to share positive as well as negative feelings (see e.g., Rose, 2002). For instance, self-disclosure and emotional support as well as co-rumination mechanisms are more frequently reported within female than male friendships (see e.g., Buhrmester \& Furman, 1987; Rose, 2002; Rose \& Rudolph, 2006).

Thus, on the one hand, socialization processes might reflect the supportive nature of female friendships. Emotional support between friends might increase friends' similarity by reducing the level of disparity between dyad members. On the other hand, co-rumination could be a mechanism underlying socialization processes that contribute to an increase in depressive symptoms within female friendships. Indeed, the only study that longitudinally examined the relationship between co-rumination and depressive symptoms found that corumination predicted depressive symptoms for female adolescents only (Rose et al., 2007). These results have important practical implications because they suggest that not only negative peer experiences (e.g., victimization, rejection) but also positive peer experiences (e.g., close friendships) may pose risks for the development of depressive symptoms (Prinstein, 2007; Rose, 2002).

The last goal of this study was to investigate whether friends' similarity and socialization processes were different for true best friends and class best friends. Findings revealed that, although socialization processes appeared to exist in female friendships for both class best friends and true best friends, they contributed to the increase in similarity between dyad members only when dyad members were the true best friends. In male friendships, socialization processes affected only the true best friends, whereas class best friends seemed to affect depressive symptoms in the opposite direction, by increasing their dissimilarity over time. Due to the lack of variables in our data set that could serve as underlying third variables, we were not able to interpret this unexpected result. Further research and replications, including additional factors (e.g., social status) that might explain such a finding, are warranted. Overall, these findings indicate that true best friends may be most influential, because these friends are more likely to share their feelings within close and intimate relationships. In addition, results indicated that socialization processes related to depressive symptoms might occur differently in male and female adolescents. Indeed, because the probability of having a true best friend who attended the same class was similar for male and female adolescents, the fact that socialization processes emerged in both female groups but only in male true best friends group might suggest that among female adolescents, socialization processes occur even in friendships that are not the best. Thus, female adolescents might be either more likely to have closer friendships compared with male adolescents or to share their feelings even with friends who are not the best (see e.g., Rose, 2002).

Our findings on true best friends have important methodological implications for future research. Friendship studies should take into account the limitations inherent in the identification of best friendships within the school setting. Specifically, future research needs to consider that when friendships are identified within schools, they are able to capture only some adolescent relations. Because adolescents are involved in salient friendships that may be established outside the school (see Vandell et al., 2005), school friendships might not represent the most intimate, close, and influential adolescent friendships. Thus, focusing exclusively on school friendships and neglecting other social contexts may provide only limited insight on adolescent friendships (Van Zalk et al., 2010).

Overall, our findings indicated that similarity in depressive symptoms occurs particularly among female adolescents and in close and intimate friendships. In addition, such similarity 
appeared to be the consequence of friends' socialization because adolescents did not seem to select peers with levels of depressive symptoms similar to their own. These findings may be interpreted in the framework of interpersonal theories of depression and models of genderspecific peer socialization (see e.g., Rose \& Rudolph, 2006). In the past, these theories have emphasized the role of difficult peer relationships in the development of depressive symptoms during adolescence (see Rudolph, Flynn, \& Abaied, 2008). Findings from this study suggest that it may be necessary for interpersonal theories of depression to focus on positive friendships as well, because adolescents may increase and exacerbate their levels of depressive symptoms in such relations. Specifically, due to the close and intimate nature of their friendships, in which they intensively share their feelings, female adolescents may be more at risk for developing depressive symptoms when interacting with their friends. The picture may be more complex for male adolescents, for whom different mechanisms (e.g., social status) may play a central role in affecting socialization processes related to depressive symptoms. In terms of practical implications, the results suggest that adolescents' intimate friendships might reflect a salient context on which preventive interventions should focus.

This study had a number of strengths, because it examined different groups of friendships, which allowed us to differentiate selection and socialization processes; utilized a dyadic approach; made a distinction between class best friends and true best friends; and utilized self-reported measures from both adolescents and their friends. Nevertheless, some limitations of this study need to be acknowledged. First, our analysis did not allow us to understand how socialization processes actually take place. In other words, we do not know whether adolescents who scored higher in depressive symptoms influenced adolescents who scored lower in depressive symptoms, or vice versa. Nevertheless, our findings on socialization effects within dyads, along with the stability of depressive symptoms over time in enduring friendships, suggest that both paths may occur. Thus, to implement preventive interventions, further research needs to investigate under which specific conditions socialization processes may lead to an increase in depressive symptoms within dyads and, conversely, under what circumstances they may protect adolescents from developing depressive symptoms.

Second, the underlying mechanisms of friends' socialization were not examined. We suggest that co-rumination may be one of the possible mechanisms, but this needs to be tested, and additional mechanisms need to be taken into account (e.g., excessive reassurance seeking). Third, limitations related to the generality of the findings arise from the nature of our sample. Attrition analyses showed that adolescents with lower levels of depressive symptoms and with some specific sociodemographic characteristics (i.e., Dutch origin, intact families) were overrepresented in the longitudinal sample. Moreover, compared with the general Dutch population, our sample included adolescents who were more highly educated, and because information on parent socioeconomic status was not available, we do not know whether our sample is representative of the national population.

Thus, these findings need to be replicated in a more representative sample of the general Dutch population as well as among adolescents residing outside the Netherlands and ethnic minority adolescents, for whom these processes might be different. In addition, it would be important to investigate whether these findings may generalize to clinical populations.

Indeed, peer relationships may be particularly difficult for clinically depressed adolescents, and socialization and selection processes may have a different meaning to them.

Finally, although this study extended previous literature on in-school friendships by distinguishing between class best friends and true best friends, the operationalization of true best friends may still be somewhat limited. Our design did not allow us to identify true best 
friend dyads outside the class. This group of friends might be different from true best friend dyads inside the class and might affect adolescent depressive symptoms differently. It may be that true best friends outside the class are most influential because they have been selected as friends for their desired characteristics (Kiesner, Poulin, \& Nicotra, 2003; Van Zalk et al., 2010). Future research is warranted to test out-of-school friendships, as well as replicate our results in larger samples.

Despite these limitations, our study provided evidence for depression socialization processes especially in female friends' dyads. These findings suggest that socialization processes may represent one of several factors attempting to explain depressive symptoms during adolescence. The fact that positive friendship relations may increase the risk for the onset and development of depressive symptoms warrants further study of friendships and internalizing behaviors.

\section{Acknowledgments}

This study was financed by the Netherlands Organization for Scientific Research Veni Grant 451-05-013.

\section{References}

Adams RE, Bukowski WM, Bagwell C. Stability of aggression during early adolescence as moderated by reciprocated friendship status and friend's aggression. International Journal of Behavioral Development. 2005; 29:139-145. doi:10.1080/01650250444000397.

Angold A, Erkanli A, Silberg J, Eaves L, Costello EJ. Depression scale scores in 8-17-year-olds: Effects of age and gender. Journal of Child Psychology and Psychiatry. 2002; 43:1052-1063. doi: 10.1111/1469-7610.00232. [PubMed: 12455926]

Berndt, TJ. Obtaining support from friends during childhood and adolescence. In: Belle, D., editor. Children's social networks and social supports. Wiley; New York, NY: 1989. p. 308-331.

Bouma, J.; Ranchor, AV.; Sanderman, R.; van Sonderen, E. Het meten van symptomen van depressie met de CES-D. University of Groningen, Northern Centre for Health-Care Research; Groningen, the Netherlands: 1995. [The measurement of symptoms of depression with the CES-D] [Internal report]

Buhrmester D, Furman F. The development of companionship and intimacy. Child Development. 1987; 58:1101-1113. doi:10.2307/1130550. [PubMed: 3608659]

Bukowski, WM.; Newcomb, AF.; Hartup, WW., editors. The company they keep: Friendship in childhood and adolescence. Cambridge University Press; New York, NY: 1996.

Byrne, D. The attraction paradigm. Academic Press; New York, NY: 1971.

Calmes CA, Roberts JE. Rumination in interpersonal relationships: Does co-rumination explain gender differences in emotional distress and relationship satisfaction among college students? Cognitive Therapy and Research. 2008; 32:577-590. doi:10.1007/s10608-008-9200-3.

CBS. Statistisch Jaarboek. Centraal Bureau voor de Statistiek; The Hague, the Netherlands: 2006. Annual report on statistics

Cillessen AHN, Jiang XL, West TV, Laszkowski DK. Predictors of dyadic friendship quality in adolescence. International Journal of Behavioral Development. 2005; 29:165-172. doi: 10.1080/01650250444000360.

Coates D, Winston T. Counteracting the deviance of depression: Peer support groups for victims. Journal of Social Issues. 1983; 39:169-194. doi:10.1111/j.1540-4560.1983.tb00147.x.

Colarossi LG, Eccles JS. Differential effects of support providers on adolescents' mental health. Social Work Research. 2003; 27:19-30.

Cook WL, Kenny DA. The actor-partner interdependence model: A model of bidirectional effects in developmental studies. International Journal of Behavioral Development. 2005; 29:101-109. doi: 10.1080/01650250444000405.

Coyne JC. Toward an interactional description of depression. Psychiatry: Journal for the Study of Interpersonal Processes. 1976; 39:28-40. 
Cuijpers P, Boluijt P, van Straten A. Screening of depression in adolescents through the Internet: Sensitivity and specificity of two screening questionnaires. European Child \& Adolescent Psychiatry. 2008; 17:32-38. doi:10.1007/s00787-007-0631-2. [PubMed: 17876508]

Dishion TJ, Andrews DW, Crosby L. Antisocial boys and their friends in early adolescence: Relationship characteristics, quality, and interactional process. Child Development. 1995; 66:710722. [PubMed: 7789197]

Engels RCME, Knibbe RA, Drop MJ, de Haan YT. Homogeneity of cigarette smoking within peer groups: Influence or selection? Health Education \& Behavior. 1997; 24:801-811. [PubMed: 9408792]

Ennett ST, Bauman KE. The contribution of influence and selection to adolescent peer group homogeneity: The case of adolescent cigarette smoking. Journal of Personality and Social Psychology. 1994; 67:653-663. doi:10.1037/0022-3514.67.4.653. [PubMed: 7965611]

Galambos NL, Leadbeater BJ, Barker ET. Gender differences in and risk factors for depression in adolescence: A 4-year longitudinal study. International Journal of Behavioral Development. 2004; 28:16-25. doi:10.1080/01650250344000235.

Garrison CZ, Addy CL, Jackson KL, McKeown RE, Waller JL. The CES-D as a screen for depression and other psychiatric disorders in adolescents. Journal of the American Academy of Child \& Adolescent Psychiatry. 1991; 30:636-641. doi:10.1097/00004583-199107000-00017. [PubMed: 1890099]

Harter, S. The development of self-representations. In: Damon, W.; Eisenberg, N., editors. Handbook of Child Psychology: Vol. 3. Social, emotional, and personality development. 5th ed.. Wiley; New York, NY: 1997. Series Ed.Vol. Ed.

Haselager GJT, Hartup WW, van Lieshout CFM, Riksen-Walraven JMA. Similarities between friends and nonfriends in middle childhood. Child Development. 1998; 69:1198-1208.

Hogue A, Steinberg L. Homophily of internalized distress in adolescent peer groups. Developmental Psychology. 1995; 31:897-906. doi: 10.1037/0012-1649.31.6.897.

Joiner TE. Contagious depression: Existence, specificity to depressed symptoms, and the role of reassurance seeking. Journal of Personality and Social Psychology. 1994; 67:287-296. doi: 10.1037/0022-3514.67.2.287. [PubMed: 7932064]

Joiner TE, Barnett J. A test of interpersonal theory of depression in children and adolescents using projective technique. Journal of Abnormal Child Psychology. 1994; 22:595-609. doi:10.1007/ BF02168940. [PubMed: 7822631]

Kandel DB. Similarity in real-life adolescent friendship pairs. Journal of Personality and Social Psychology. 1978; 36:306-312. doi: 10.1037/0022-3514.36.3.306.

Kashy, DA.; Kenny, DA. The analysis of data from dyads andgroups. In: Reis, HT.; Judd, CM., editors. Handbook of research methods in social and personality psychology. Cambridge University Press; New York, NY: 2000. p. 451-477.

Kelley, HH.; Thibaut, JW. Interpersonal relations: A theory of interdependence. Wiley; New York, NY: 1978.

Kenny DA. The effect of nonindependence on significance testing in dyadic research. Personal Relationships. 1995; 2:67-75. doi: 10.1111/j.1475-6811.1995.tb00078.x.

Kenny, DA.; Kashy, DA.; Cook, W. Dyadic data analysis. Guilford Press; New York, NY: 2006.

Kiesner J, Poulin F, Nicotra E. Peer relations across contexts: Individual-network homophily and network inclusion in and after school. Child Development. 2003; 74:1328-1343. doi: 10.1111/1467-8624.00610. [PubMed: 14552401]

La Greca AM, Harrison HM. Adolescent peer relations, friendships, and romantic relationships: Do they predict social anxiety and depression? Journal of Clinical Child and Adolescent Psychology. 2005; 34:49-61. doi:10.1207/s15374424jccp3401_5. [PubMed: 15677280]

Larsen JK, Otten R, Engels RCME. Adolescent depressive symptoms and smoking behavior: The gender-specific role of weight concern and dieting. Journal of Psychosomatic Research. 2009; 66:305-308. doi:10.1016/j.jpsychores.2008.10.006. [PubMed: 19302887]

Laursen, B.; Popp, D.; Burk, WJ.; Kerr, M.; Stattin, H. Incorporating interdependence into developmental research: Examples from the study of homophily and homogeneity. In: Card, NA.; 
Selig, JP.; Little, TD., editors. Modeling dyadic and interdependent data in developmental research. Erlbaum; Mahwah, NJ: 2008. p. 11-38.

McPherson M, Smith-Lovin L, Cook JM. Birds of a feather: Homophily in social networks. Annual Review of Sociology. 2001; 27:415-444. doi:10.1146/annurev.soc.27.1.415.

Muthén, LK.; Muthén, BO. Mplus user's guide. 5th ed.. Muthén \& Muthén; Los Angeles, CA: 1998-2007.

Nolan SA, Flynn C, Garber J. Prospective relations between rejection and depression in young adolescents. Journal of Personality and Social Psychology. 2003; 85:745-755. doi: 10.1037/0022-3514.85.4.745. [PubMed: 14561127]

Olsen JA, Kenny DA. Structural equation modeling with interchangeable dyads. Psychological Methods. 2006; 11:127-141. doi: 10.1037/1082-989X.11.2.127. [PubMed: 16784334]

Parker JG, Asher SR. Friendship and friendship quality in middle childhood: Links with peer group acceptance and feelings of loneliness and social dissatisfaction. Developmental Psychology. 1993; 29:611-621. doi:10.1037/0012-1649.29.4.611.

Popp D, Laursen B, Kerr M, Stattin H, Burk WJ. Modeling homophily over time with an actor-partner interdependence model. Developmental Psychology. 2008; 44:1028-1039. doi: 10.1037/0012-1649.44.4.1028. [PubMed: 18605832]

Poulin F, Boivin M. The role of proactive and reactive aggression in the formation and development of boys' friendships. Developmental Psychology. 2000; 36:233-240. doi: 10.1037/0012-1649.36.2.233. [PubMed: 10749080]

Prinstein MJ. Moderators of peer contagion: A longitudinal examination of depression socialization between adolescents and their best friends. Journal of Clinical Child and Adolescent Psychology. 2007; 36:159-170. doi:10.1080/15374410701274934. [PubMed: 17484689]

Prinstein MJ, Boergers J, Vernberg EM. Overt and relational aggression in adolescence: Socialpsychological adjustment of aggressors and victims. Journal of Clinical Child Psychology. 2001; 30:479-491. doi:10.1207/S15374424JCCP3004_05. [PubMed: 11708236]

Roberts RE, Lewinsohn PM, Seeley JR. Screening for adolescent depression: A comparison of depression scales. Journal of the American Academy of Child \& Adolescent Psychiatry. 1991; 30:58-66. doi:10.1097/00004583-199101000-00009. [PubMed: 2005065]

Rose AJ. Co-rumination in the friendships of girls and boys. Child Development. 2002; 73:1830-1843. doi:10.1111/1467-8624.00509. [PubMed: 12487497]

Rose AJ, Carlson W, Waller EM. Prospective associations of co-rumination with friendship and emotional adjustment: Considering the socioemotional trade-offs of co-rumination. Developmental Psychology. 2007; 43:1019-1031. doi:10.1037/0012-1649.43.4.1019. [PubMed: 17605532]

Rose AJ, Rudolph KD. A review of sex differences in peer relationship processes: Potential trade-offs for the emotional and behavioral development of girls and boys. Psychological Bulletin. 2006; 132:98-131. doi:10.1037/0033-2909.132.1.98. [PubMed: 16435959]

Rosenblatt A, Greenberg J. Examining the world of the depressed: Do depressed people prefer others who are depressed? Journal of Personality and Social Psychology. 1991; 60:620-629. doi: 10.1037/0022-3514.60.4.620. [PubMed: 2037970]

Rudolph, KD.; Flynn, M.; Abaied, JL. A developmental perspective on interpersonal theories of youth depression. In: Abela, JRZ.; Hankin, BL., editors. Handbook of depression in children and adolescents. Guilford Press; New York, NY: 2008. p. 79-102.

Satorra A, Bentler PM. A scaled difference chi-square test statistic for moment structure analysis. Psychometrika. 2001; 66:507-514. doi:10.1007/BF02296192.

Slavin LA, Rainer KL. Gender differences in emotional support and depressive symptoms among adolescents: A prospective analysis. American Journal of Community Psychology. 1990; 18:407421. doi:10.1007/BF00938115. [PubMed: 2264557]

Snijders, TAB.; Steglich, C.; Schweinberger, M. Modeling the co-evolution of networks and behavior. In: Van Montfort, K.; Oud, H.; Satorra, A., editors. Longitudinal models in the behavioral and related sciences. Erlbaum; Mahwah, NJ: 2007. p. 41-71.

Stevens EA, Prinstein MJ. Peer contagion of depressogenic attributional styles among adolescents: A longitudinal study. Journal of Abnormal Child Psychology. 2005; 33:25-37. doi:10.1007/ s10802-005-0931-2. [PubMed: 15759589] 
Strack S, Coyne JC. Social confirmation of dysphoria: Shared and private reactions to depression. Journal of Personality and Social Psychology. 1983; 44:798-806. doi: 10.1037/0022-3514.44.4.798. [PubMed: 6842366]

Vandell, DL.; Pierce, KM.; Dadisman, K. Out-of-school setting as a developmental context for children and youth. In: Kail, R., editor. Advances in child development and behaviour. Vol. 33. Academic; New York, NY: 2005. p. 43-77.

Van Zalk MHV, Kerr M, Branje SJT, Stattin H, Meeus WHJ. It takes three: Selection, influence, and de-selection processes of depression in adolescent friendship networks. Developmental Psychology. 2010; 46:927-938. doi:10.1037/a0019661. [PubMed: 20604612]

Vernberg EM. Psychological adjustment and experiences with peers during early adolescence: Reciprocal, incidental, or unidirectional relationships? Journal of Abnormal Child Psychology. 1990; 18:187-198. doi:10.1007/BF00910730. [PubMed: 2348032]

Wade TJ, Cairney J, Pevalin DJ. Emergence of gender differences in depression during adolescence: National panel results from three countries. Journal of the American Academy of Child \& Adolescent Psychiatry. 2002; 41:190-198. doi:10.1097/00004583-200202000-00013. [PubMed: 11837409] 


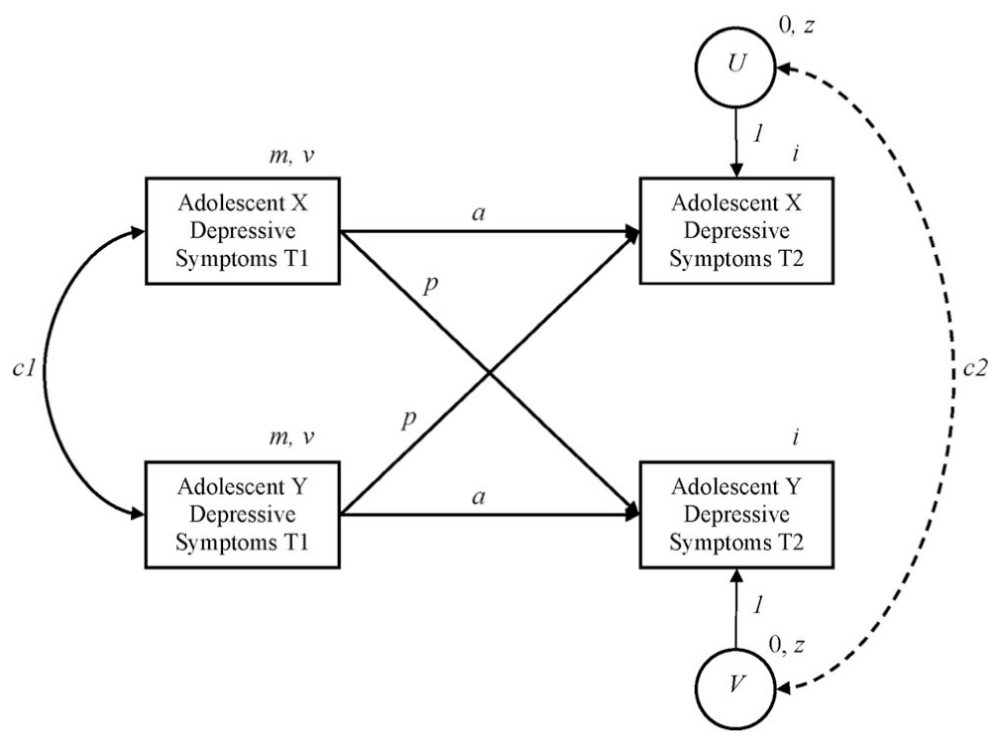

Figure 1.

Longitudinal actor-partner interdependence model for depressive symptoms between indistinguishable best friend dyads. $a=$ actor effects; $p=$ partner effects; $c 1=$ concurrent correlation between adolescent $X$ 's depressive symptoms and adolescent $Y$ 's depressive symptoms at Time 1 (T1); $U=$ residual variance in adolescent X's depressive symptoms at Time 2 (T2); $V=$ residual variance in adolescent Y's depressive symptoms at Time 2; $c 2=$ correlation between adolescent $\mathrm{X}$ 's depressive symptoms residual $(U)$ and adolescent $\mathrm{Y}$ 's depressive symptoms residual $(V)$ at Time $2 ; m=$ predictor means; $V=$ predictor variances; $i$ $=$ outcome intercepts; $z=$ residual variances. 


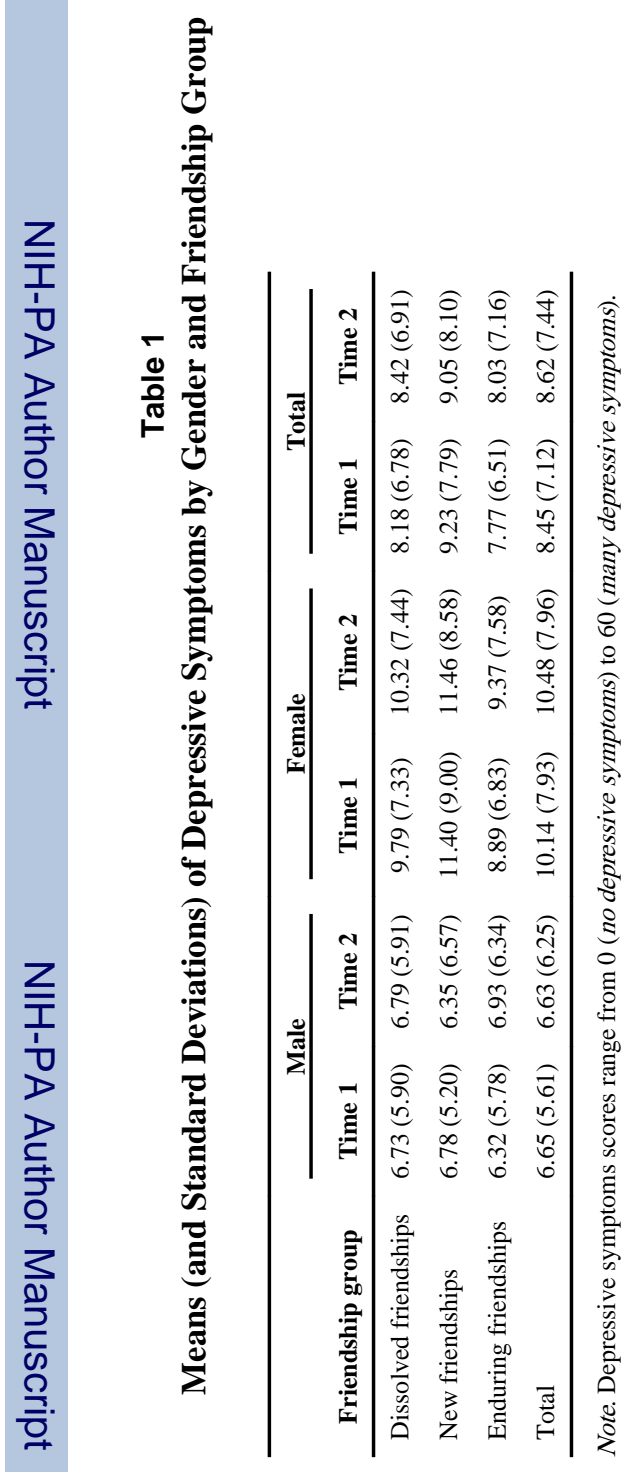




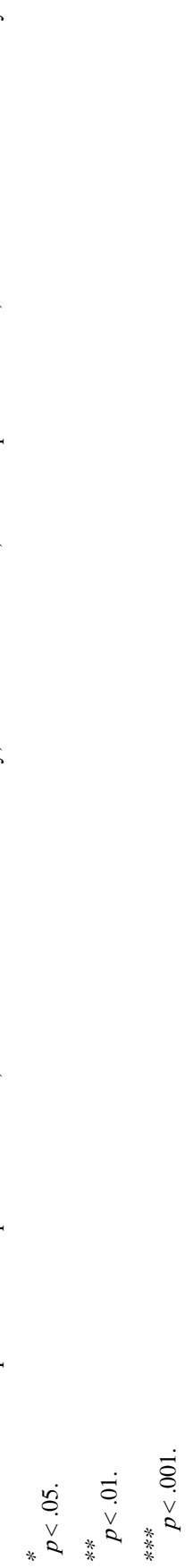




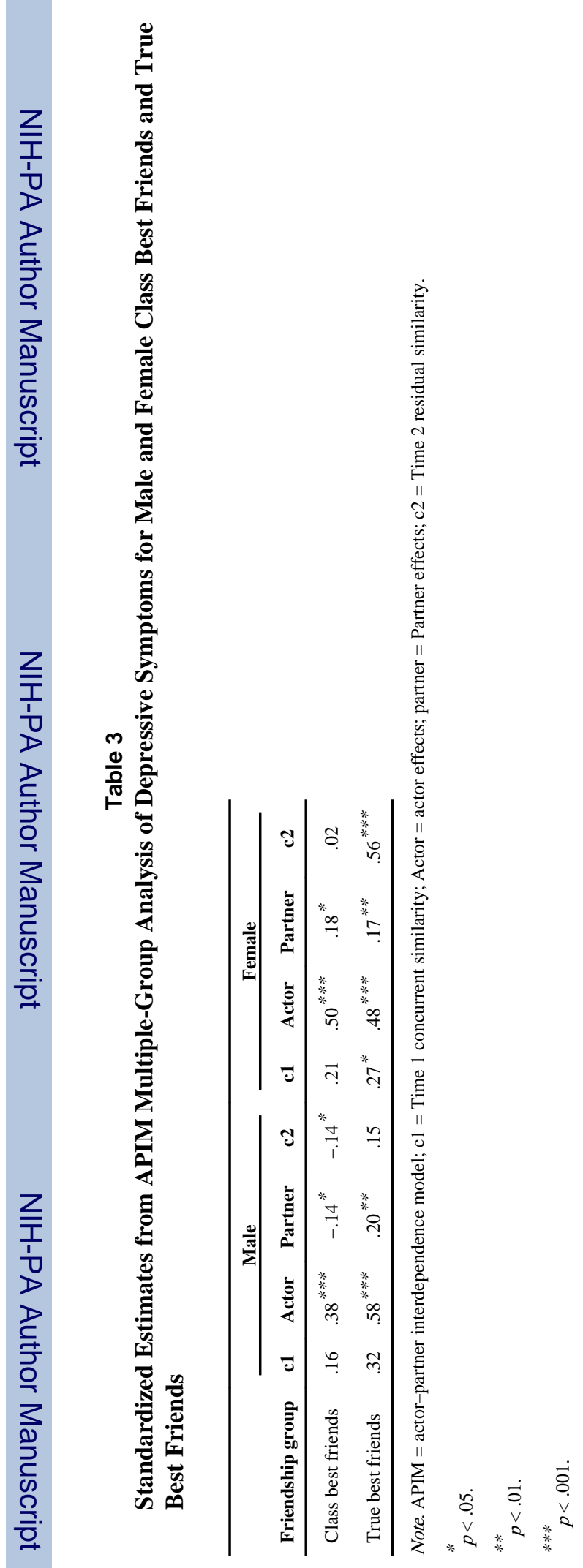

